\title{
Update on the assessment and investigation of adult obstructive sleep apnoea
}

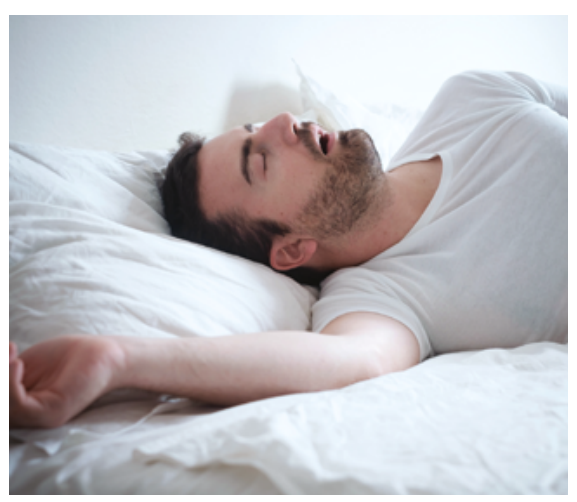

CPD

Garun S Hamilton,

Ching Li Chai-Coetzer

\section{Background}

Obstructive sleep apnoea (OSA) is common. Medicare Benefits Schedule rules regarding which patients are eligible for a sleep study without first needing to see a sleep or respiratory specialist have recently changed and incorporate validated questionnaires of OSA risk and subjective sleepiness.

\section{Objective}

The aim of this article is to bring general practitioners (GPs) up to date with the key factors that should be assessed when considering whether a patient has OSA. It also highlights the strengths and weaknesses of the screening questionnaires, and the pros and cons of different types of sleep studies.

\section{Discussion}

OSA may significantly affect quality of life, mood, safety and cardiovascular risk. Assessment should focus on symptoms. Screening questionnaires have high sensitivity but, when used alone, poor specificity for moderate-tosevere OSA. The Epworth Sleepiness Scale (ESS) is a poor marker of OSA but does predict response to treatment when elevated. GPs can directly order sleep studies when OSA questionnaires are positive and the ESS is elevated; however, negative questionnaires do not exclude OSA or another sleep disorder. obstructive SLEep APNOEA (OSA) is the repetitive collapse of the upper airway during sleep. The collapse is either complete, leading to cessation of airflow (apnoea), or partial, leading to a reduction in airflow (hypopnoea). Partial collapse is more common. The severity of OSA is usually determined by the frequency of obstructive respiratory events and defined by the Apnoea Hypopnoea Index (AHI), which is the average number of respiratory disturbances per hour of sleep. OSA has been arbitrarily defined as an $\mathrm{AHI} \geq 5$ events/hour, and moderate-to-severe OSA is defined as an AHI $\geq 15$ events/ hour. ${ }^{1}$ The prevalence of OSA is very high in the general adult population, with figures as high as $38 \%$, depending on the population studied and definition used..$^{2}$ Much of this OSA is mild in severity (AHI between 5-15 events/ hour) and minimally symptomatic. Although not associated with adverse vascular sequelae, mild OSA may still be associated with troublesome daytime symptoms in some subjects. Moderateto-severe OSA (AHI $\geq 15$ events/ hour) is more commonly symptomatic and is associated with other adverse outcomes such as cardiovascular and cerebrovascular disease, cognitive decline, motor vehicle accidents and depression. It is also very common, being found in $6-17 \%$ of the general adult population, with a recent Australian study showing a prevalence of $8.3 \% .^{3,4}$ In general, both mild OSA plus daytime symptoms and moderate-to-severe OSA are indications to consider OSA treatment.

Given the high prevalence of OSA in the general adult population, a large number of patients presenting to general practitioners (GPs) will have OSA. Known risk factors include obesity, adenotonsillar hypertrophy (mainly seen in children and young adults), increasing age, type 2 diabetes, alcohol and sedative use, and hypertension that is difficult to control. ${ }^{5,6}$ Because many patients with OSA may be minimally symptomatic, it is unclear whether there are overall benefits to widespread screening of either the general population or high-risk individuals (eg those with type 2 diabetes). A recent review of the literature by the US Preventive Services Task Force has concluded that there is insufficient evidence to support screening for OSA in otherwise asymptomatic individuals. ${ }^{7,8}$ Nevertheless, a large number of patients presenting to GPs will have symptoms of OSA, and it is important to consider 'trigger symptoms' that warrant further assessment of OSA risk and determination of the need for sleep testing. 


\section{OSA symptoms}

Adult patients with OSA typically present to GPs for at least one of three major reasons, and these reasons commonly overlap within a patient. First, they may be concerned about their own daily symptoms. These include unrefreshing sleep, tiredness and fatigue, poor concentration and focus, a low mood or excessive daytime sleepiness. Second, they may be suspicious that they have OSA (eg because of apnoeas witnessed by their partner) and are concerned about the potential adverse health consequences. This may include immediate problems such as safety driving or worry about more long-term concerns such as cardiovascular disease, stroke or cognitive decline. Finally, their concerns may relate more to the effect on their bed partner. Socially disruptive snoring with or without witnessed apnoeic episodes is a common initial trigger symptom that should lead to questioning about the other symptoms listed above. It is important to note that OSA in young children presents differently to OSA in adults. Although snoring, witnessed apnoeas and daytime sleepiness may be present, sleepiness is less common than in adults. It is important to consider inattention, irritability and behavioural and learning problems as presenting features. The rest of this article refers to adult OSA, not paediatric OSA.

\section{Clinical assessment}

As well as asking about symptoms related to OSA in the clinical assessment, it is important to consider a number of other factors that may affect the diagnostic and treatment pathway. These include the following.

- Signs of adenotonsillar hypertrophy. Although less common with increasing age, this is important as it points to a potential surgical treatment for OSA.

- Driving and workplace safety. If OSA is suspected in commercial drivers or other safety-critical workers - or if there are symptoms of sleepiness while driving - assessment for OSA (or another sleep disorder) should be undertaken urgently and advice about driving risk provided. ${ }^{9}$
- Obesity. This is the major reversible risk factor for OSA, and OSA symptoms commonly develop in conjunction with a period of rapid weight gain. ${ }^{10}$ This is an opportune time to address the obesity and its associated cardiometabolic risks.

- Hypertension. OSA is associated with hypertension, and continuous positive airway pressure (CPAP) treatment may improve blood pressure control, particularly if the OSA is severe or the hypertension is treatmentresistant. ${ }^{6,11}$

- Depression. This commonly co-exists with OSA and shares similar symptoms of tiredness, fatigue, sleepiness and poor motivation. Depression is also a risk factor for an incomplete response to CPAP treatment and may need to be independently addressed.

\section{OSA screening questionnaires}

\section{Berlin Questionnaire}

The first screening questionnaire for OSA that was designed specifically for use in general practice was the Berlin Questionnaire (BQ). ${ }^{12}$ The BQ was a consensus outcome following a conference on sleep in primary care held in Berlin, Germany, in 1996 that was attended by US and German respiratory and primary care physicians. The BQ consists of 11 items within three different categories that ask about:

- snoring and witnessed apneas (category 1)

- daytime sleepiness or fatigue (category 2)
- self-reported hypertension and obesity (body mass index [BMI]; category 3). Patients are then classified as either high risk or low risk for OSA. In a meta-analysis of BQ performance for predicting a diagnosis of moderateto-severe OSA (defined as an $\mathrm{AHI} \geq 15$ events/hour), there was a sensitivity (ie proportion of patients with OSA who had a positive test) of $82 \%$, and a specificity (ie proportion of patients without OSA who had a negative test) of $39 \% .^{13}$

\section{OSA50 questionnaire}

The OSA50 questionnaire (Table 1) was developed by a group of Australian sleep medicine researchers who aimed to create a brief, four-item OSA screening tool for use in general practice. ${ }^{14}$ They established that the four items that best predicted a diagnosis of severe OSA (ie $\mathrm{AHI} \geq 30$ events/hour) were:

1. obesity (by waist circumference)

2. snoring

3. witnessed apneas

4. age $\geq 50$ years.

Weightings from results of logistic regression analyses were used to create a simple screening tool with a maximum score of 10 points. An OSA 50 score $\geq 5$ points was found in a validation sample to have $94 \%$ sensitivity, with $31 \%$ specificity for identifying moderate-to-severe OSA.

\section{STOP-BANG questionnaire}

The STOP-BANG questionnaire (www. stopbang.ca/osa/screening.php) was developed by an anaesthetist from Toronto, Canada, and was originally validated for use in a pre-operative,

\section{Table 1. OSA50 questionnaire}

\begin{tabular}{llr}
\hline & & If yes, score \\
\hline Obesity & Waist circumference* $(>102 \mathrm{~cm}$ for males or $>88 \mathrm{~cm}$ for females $)$ & 3 \\
\hline Snoring & Has your snoring ever bothered other people? & 3 \\
\hline Apnoeas & Has anyone noticed that you stop breathing during your sleep? & 2 \\
\hline $\mathbf{5 0}$ & Are you aged 50 years or over? & 2 \\
\hline & Total score & $\ldots . . . . / \mathbf{1 0}$ points
\end{tabular}

*Waist measurement to be measured at the level of the umbilicus (males $102 \mathrm{~cm}=$ size 40 [inches] and females $88 \mathrm{~cm}=$ size 16) 
elective surgery population. ${ }^{15}$ It consists of eight yes/no questions related to:

1. snoring

2. tiredness

3. observed apneas

4. high blood pressure

5. $\mathrm{BMI}>35 \mathrm{~kg} / \mathrm{m}^{2}$

6. age $>50$ years

7. neck circumference $>40 \mathrm{~cm}$

8. male gender.

High risk for OSA is defined as a positive response to $\geq 3$ items. A potential advantage of the STOP-BANG questionnaire is that different cut-off scores can be used to trade off sensitivity and specificity. For example, using a higher cut-off score reduces the sensitivity but increases the specificity of the STOP-BANG questionnaire. ${ }^{16}$ This means a higher cut-off will miss more cases of OSA when negative, but a positive result will be more likely to be a true positive. This is highly relevant when considering the MBS item number rules, which specify that a cut-off score of $\geq 4$ be used.

\section{Performance of OSA screening questionnaires}

OSA screening questionnaires at recommended cut-off points tend to have high sensitivity but poor specificity (Table 2); in other words, while the majority of patients who have OSA will screen positive, there will be a large number of false-positive results. ${ }^{13,14,16}$ Therefore, screening questionnaires alone are inadequate for confirming a diagnosis, and patients who have a positive screening test should proceed to further evaluation with formal sleep study testing to confirm the presence or absence of OSA.

Surprisingly, very few studies have assessed the performance of the questionnaires in primary care populations. Ongoing Australian research suggests that the sensitivity in primary care is lower than in other selected populations. This means that a negative screening questionnaire does not rule out OSA (or any alternative sleep disorder), and referral to a sleep medicine specialist is recommended if there is ongoing doubt.

These questionnaires have not been validated in children and should only be used in adults.

\section{Epworth Sleepiness Scale}

The Epworth Sleepiness Scale (ESS) provides a subjective measure of daytime sleepiness by asking the patient to rate their chance of dozing off (score of 0-3) in eight commonly encountered scenarios to provide a score out of 24 , with higher scores indicating greater degrees of daytime sleepiness. ${ }^{17}$ An ESS score $\geq 8$ suggests the presence of at least mild daytime sleepiness. ${ }^{18}$ Of critical importance, the ESS score does not correlate well with AHI, as not all patients with OSA will have symptoms of sleepiness detected by the ESS and there are multiple causes for excessive daytime sleepiness beyond OSA (eg other sleep disorders, depression). Therefore, the ESS should not be used alone as a screening tool to identify patients at high risk of OSA. When used in conjunction with an
OSA screening questionnaire, the ESS is likely to be helpful in identifying patients at high risk of symptomatic disease who will benefit from treatment. However, this remains the subject of ongoing research and it is important to realise that the majority of patients with OSA will not have an ESS $\geq 8 .{ }^{19,20}$

\section{Medicare Benefits Schedule rules regarding primary care referral pathways for sleep studies}

The new Medicare Benefits Schedule (MBS) rules have resulted in two potential pathways to a sleep study.

1. GPs may refer patients to a sleep or respiratory medicine specialist for a clinical assessment; the specialist can then order a sleep test as appropriate.

2. GPs may directly order a sleep test (either at home or in a sleep laboratory), without the patient first needing a clinical review by a sleep or respiratory specialist, if certain criteria are met. Patients must have a positive OSA screening questionnaire (either BQ positive, OSA $50 \geq 5$ or a STOP-BANG cut-off of $\geq 4$ ), in addition to having an ESS $\geq 8$. The aim is to allow GPs to expedite diagnosis and treatment in a highly symptomatic group of patients with OSA; however, it is important to realise that many patients with significant OSA will not meet this questionnaire combination. These patients will need assessment by sleep or respiratory medicine specialists. Refer to Figure 1 for potential investigation pathways.

Table 2. Diagnostic utility of obstructive sleep apnoea screening questionnaires for detecting moderate-to-severe obstructive sleep apnoea

\begin{tabular}{|c|c|c|c|c|}
\hline & Berlin Questionnaire ${ }^{13}$ & STOP-BANG cut-off $\geq 3^{16}$ & STOP-BANG cut-off $\geq 4^{16}$ & OSA50 $^{14}$ \\
\hline Sensitivity* \% & 82 & 94 & 81 & 94 \\
\hline Specificity $\%$ & 39 & 32 & 51 & 31 \\
\hline
\end{tabular}

Data taken from validation studies ${ }^{14}$ and meta-analyses ${ }^{13,16}$

*Sensitivity refers to the proportion of subjects with OSA who have a positive questionnaire. (Note: for a highly sensitive test, a negative result is good at ruling out disease.)

'Specificity refers to the proportion of subjects without OSA who have a negative questionnaire. (Note: for a highly specific test, a positive result is good at ruling in disease.)

In general, OSA screening questionnaires have good but not optimal sensitivity, and poor specificity for detecting AHI $\geq 15$ events/hour. OSA, obstructive sleep apnoea; AHI, Apnoea Hypopnoea Index 


\section{Types of sleep studies}

The gold standard test for diagnosing OSA is with in-laboratory full polysomnography (PSG) with a sleep technician in attendance throughout the night. However, in-laboratory PSG is limited in its availability, labour intensive and costly, so increasing attention has been focused on home sleep study testing.

Traditionally, sleep studies have been divided into the following four categories:

- Type 1: Attended, in-laboratory, full PSG with $\geq 7$ recording channels measuring sleep stage, breathing and cardiac parameters, and limb movements.

- Type 2: Unattended, home, full PSG with $\geq 7$ recording channels.

- Type 3: Limited channel monitoring of breathing parameters without sleep assessment.
Questionnaire negative, ESS $\geq 8$

Consider other sleep disorders, mood disorders and sleep specialist referral.

\section{Questionnaire positive, ESS $<8$}

OSA still possible, consider referral to sleep specialist.
Patient presents with symptoms suspicious for OSA.

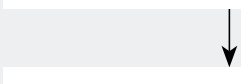

Assessment of further history, examination, comorbidities, driving risk.

Refer to sleep specialist
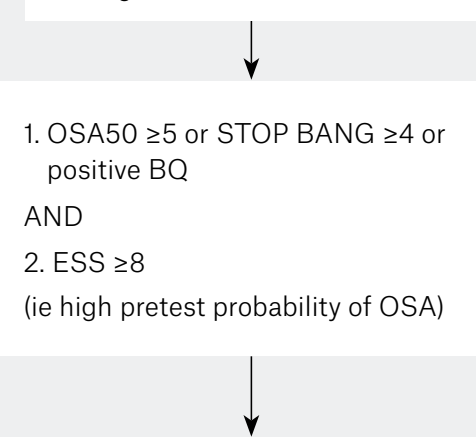

Direct referral for diagnostic PSG:

- Level 2 home or Level 1 in-lab where home study not suitable.

- Level 3 or 4 study may be acceptable depending on local resources (eg rural setting).

- ASA/NATA-accredited laboratory recommended but not mandatory.

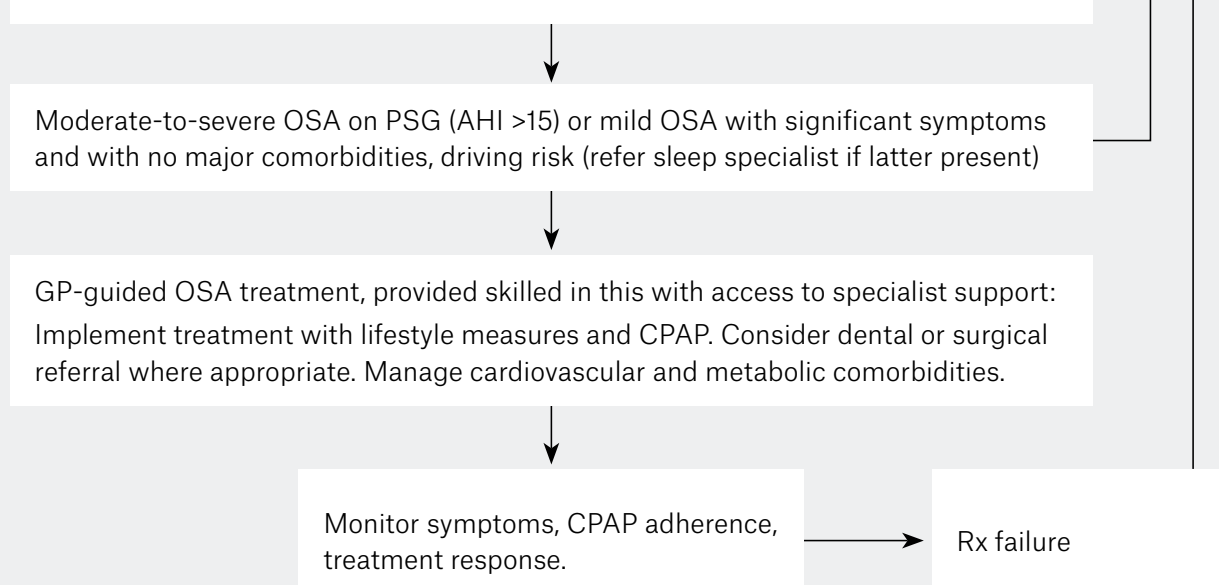

Figure 1. Potential flow chart for OSA investigation

ASA, Australasian Sleep Association; BQ, Berlin Questionnaire; CPAP, continuous positive airway pressure; ESS, Epworth Sleepiness Scale; GP, general practitioner; NATA, National Association of Testing Authorities, Australia; OSA, obstructive sleep apnoea; Rx, treatment 
- Type 4: Limited channel monitoring of only 1-2 channels (eg oximetry). Results from home PSG (type 2) testing have been shown to have a high level of agreement when compared with in-laboratory PSG for the diagnosis of OSA, although there is greater potential for signal loss and study failure ( 7\%) due to the lack of an attending sleep technician. ${ }^{21,22}$ While home PSG testing is likely to be appropriate for many patients with a suspected diagnosis of OSA, some patients should undergo attended, in-laboratory (type 1) PSG testing. ${ }^{23}$ Factors that may make home sleep studies unsuitable for some patients include the following:

\section{Patient-related factors}

1. Neuropsychological (eg severe intellectual disability, neuromuscular disease, major communication difficulties)

2. Severe physical disability with inadequate carer attendance

3. Unsuitable home environment (eg noise levels, family interactions, distance from sleep laboratory, staff safety concerns)

4. Discretionary (eg symptoms or former test results do not equate with clinical impression; patients seeking a second opinion where original diagnosis is uncertain; serious medicolegal consequences are relevant; patient preference on the basis of factors such as distance to travel, cost, concern about the equipment etc)

\section{Sleep disorder-related factors}

1. Consideration of other sleep disorders such as central sleep apnoea, hypoventilation, heart failure, neurological disorders, sleep-related movement disorder, parasomnia or seizure disorder, unexplained hypersomnolence

2. Video confirmation of body positional/ rotational aspects of sleep-disordered breathing required, or of other associated movements.

There has been growing interest in the use of unattended type 3 and 4 limitedchannel sleep studies as a result of their ability to expedite OSA diagnosis and treatment and their potential to reduce healthcare costs. Type 3 and 4 studies have been shown to have good ability to confirm OSA in highly selected patients with a high pre-test probability of moderate-to-severe OSA without other significant medical or sleep comorbidities. ${ }^{24,25}$ MBS reimbursement is not currently available in Australia for type 3 and 4 testing, so their use in this country has remained limited.

\section{Conclusion}

OSA is a treatable, common disease presenting to GPs. Untreated, it may have major adverse impacts on health. An initial clinical assessment, which includes validated questionnaires, helps triage patients to sleep testing pathways that have now become embedded in the MBS. Despite their ability to recognise some patients with sleepiness and a high likelihood of OSA who can proceed to expedited sleep testing, it is important to note that negative questionnaires (particularly the ESS) do not rule out patients having clinically significant OSA. These patients will need specialist referral.

\section{Authors}

Garun S Hamilton MBBS, FRACP, PhD, Director of Sleep Medicine Research, Lung and Sleep Medicine, Monash Health, Clayton, Vic; Clinical Associate Professor, School of Clinical Sciences, Monash University, Clayton, Vic; Monash Partners - Epworth, Melbourne, Vic. garun.hamilton@monashhealth.org Ching Li Chai-Coetzer MBBS, FRACP, GCPH, PhD, Senior Postdoctoral Research Fellow, Adelaide Institute of Sleep Health, Flinders University, Bedford Park, SA; Clinical Lead, Sleep Health Service, Respiratory and Sleep Service, Southern Adelaide Local Health Network, Flinders Medical Centre, Bedford Park, SA

Competing interests: GSH has received equipment to support research from Resmed, Philips Respironics and Air Liquide Healthcare. GSH was a member of the Thoracic Medicine Clinical Committee of the MBS Review. CLC created the OSA50 questionnaire that features in this article and is a chief investigator on the NHMRC Centres of Research Excellence Project titled, 'National centre for sleep services research - Positioning primary care at the centre of sleep health management'.

Funding: None.

Provenance and peer review: Commissioned, externally peer reviewed.

\section{References}

1. Young T, Palta M, Dempsey J, Skatrud J, Weber S, Badr S. The occurrence of sleep-disordered breathing among middle-aged adults. N Engl J Med 1993;328(17):1230-35.
2. Senaratna CV, Perret JL, Lodge CJ, et al. Prevalence of obstructive sleep apnea in the general population: A systematic review. Sleep Med Rev 2017;34:70-81. doi: 10.1016/j. smrv.2016.07.002.

3. Adams RJ, Appleton SL, Taylor AW, et al. Sleep health of Australian adults in 2016: Results of the 2016 Sleep Health Foundation national survey. Sleep Health 2017;3(1):35-42. doi: 10.1016/j. sleh.2016.11.005.

4. Appleton SL, Gill TK, Lang CJ, et al. Prevalence and comorbidity of sleep conditions in Australian adults: 2016 Sleep Health Foundation national survey. Sleep Health 2018;4(1):13-19. doi: 10.1016/j. sleh.2017.10.006.

5. Young T, Skatrud J, Peppard PE. Risk factors for obstructive sleep apnea in adults. JAMA 2004;291(16):2013-16. doi: 10.1001/ jama.291.16.2013.

6. Martínez-García MA, Capote F Campos-Rodríguez F, et al. Effect of CPAP on blood pressure in patients with obstructive sleep apnea and resistant hypertension: The HIPARCO randomized clinical trial. JAMA 2013;310(22):2407-15. doi: 10.1001/ jama.2013.281250.

7. Force USPST, Bibbins-Domingo K, Grossman DC, et al. Screening for obstructive sleep apnea in adults: US Preventive Services Task Force recommendation statement. JAMA 2017;317(4):407-14. doi: 10.1001/jama.2016.20325.

8. Jonas DE, Amick HR, Feltner C, et al. Screening for obstructive sleep apnea in adults: Evidence report and systematic review for the US Preventive Services Task Force. JAMA 2017;317(4):415-33. doi: 10.1001/jama.2016.19635.

9. Ausroads. Assessing fitness to drive: For health professionals. Sydney, NSW: Ausroads, 2016. Available at https://austroads.com.au/ drivers-and-vehicles/assessing-fitness-to-drive/forhealth-professionals [Accessed 2 December 2018].

10. Phillips BG, Hisel TM, Kato M, et al. Recent weight gain in patients with newly diagnosed obstructive sleep apnea. J Hypertens 1999;17(9):1297-300.

11. Montesi SB, Edwards BA, Malhotra A, Bakker JP. The effect of continuous positive airway pressure treatment on blood pressure: A systematic review and meta-analysis of randomized controlled trials. J Clin Sleep Med 2012;8(5):587-96. doi: 10.5664/ jcsm.2170.

12. Netzer NC, Stoohs RA, Netzer CM, Clark K, Strohl KP. Using the Berlin Questionnaire to identify patients at risk for the sleep apnea syndrome. Ann Intern Med 1999;131(7):485-91.

13. Senaratna CV, Perret JL, Matheson MC, et al. Validity of the Berlin questionnaire in detecting obstructive sleep apnea: A systematic review and meta-analysis. Sleep Med Rev 2017;36:116-24. doi: 10.1016/j.smrv.2017.04.001.

14. Chai-Coetzer CL, Antic NA, Rowland LS, et al. A simplified model of screening questionnaire and home monitoring for obstructive sleep apnoea in primary care. Thorax 2011;66(3):213-19. doi: 10.1136/thx.2010.152801.

15. Chung F, Yegneswaran B, Liao P, et al. STOP questionnaire: $A$ tool to screen patients for obstructive sleep apnea. Anesthesiology 2008;108(5):812-21. doi: 10.1097/ ALN.0b013e31816d83e4.

16. Chiu HY, Chen PY, Chuang LP, et al. Diagnostic accuracy of the Berlin questionnaire, STOPBANG, STOP, and Epworth sleepiness scale in detecting obstructive sleep apnea: A bivariate meta-analysis. Sleep Med Rev 2017;36:57-70. doi: 10.1016/j.smrv.2016.10.004. 
17. Johns MW. A new method for measuring daytime sleepiness: The Epworth sleepiness scale. Sleep 1991;14(6):540-45.

18. Antic NA, Buchan C, Esterman A, et al. A randomized controlled trial of nurse-led care for symptomatic moderate-severe obstructive sleep apnea. Am J Respir Crit Care Med 2009;179(6):501-08. doi: 10.1164/rccm.20081015580C.

19. Duran J, Esnaola S, Rubio R, Iztueta A. Obstructive sleep apnea-hypopnea and related clinical features in a population-based sample of subjects aged 30 to 70 yr. Am J Respir Crit Care Med 2001;163(3 Pt 1):685-89.

20. Heinzer R, Vat S, Marques-Vidal P, et al. Prevalence of sleep-disordered breathing in the general population: The HypnoLaus study. Lancet Respir Med 2015;3(4):310-18. doi: 10.1016/S22132600(15)00043-0.

21. Campbell AJ, Neill AM. Home set-up polysomnography in the assessment of suspected obstructive sleep apnea. J Sleep Res 2011;20(1 Pt 2):207-13. doi: 10.1111/j.13652869.2010.00854.x.
22. Iber C, Redline S, Kaplan Gilpin AM, et al. Polysomnography performed in the unattended home versus the attended laboratory setting Sleep Heart Health Study methodology. Sleep 2004;27(3):536-40.

23. Douglas JA, Chai-Coetzer CL, McEvoy D, et al. Guidelines for sleep studies in adults A position statement of the Australasian Sleep Association. Sleep Med 2017;36 Suppl 1:S2-S22. doi: 10.1016/j.sleep.2017.03.019.

24. Abrahamyan L, Sahakyan Y, Chung S, et al. Diagnostic accuracy of level IV portable sleep monitors versus polysomnography for obstructive sleep apnea: A systematic review and meta-analysis. Sleep Breath 2018;22(3):593-611. doi: 10.1007/s11325017-1615-1.

25. El Shayeb M, Topfer LA, Stafinski T, Pawluk L, Menon D. Diagnostic accuracy of level 3 portable sleep tests versus level 1 polysomnography for sleep-disordered breathing: A systematic review and meta-analysis. CMAJ 2014;186(1):E25-51. doi: 10.1503/cmaj.130952. 